\title{
Mechanical Ventilation of Patients With and Without ARDS: How Far Have We Come?
}

Nearly 13 years ago, the publication of results from the ARDS Network's ARMA trial revealed an $8.8 \%$ absolute mortality reduction in patients with acute lung injury (ALI)/ ARDS receiving a low tidal volume ventilation strategy. ${ }^{1}$ One might have guessed at the time that such unambiguous benefit from a low-cost (strictly speaking, marginally no-cost) intervention, with no need for new equipment, and minimal additional training, would be embraced by clinicians and adopted widely. But such a guess would ignore the well described chasm between evidence-derived best practices and "real world" care as it is delivered. . $^{2,3}$ While much attention is paid to the need for translational research to bridge the gap between the lab bench and the patient's bedside, an equally important gap exists between our best available clinical evidence and our current clinical practice.

Indeed, post-ARMA studies of the adoption of lungprotective ventilation protocols in patients with ALI/ARDS have been discouraging, with estimates of provider adherence ranging from $39 \%$ to $57 \%$, even in recent years. ${ }^{4-8}$ Several studies have explored barriers to implementation of lung-protective ventilation, ,,5,9 identifying provider discomfort with low tidal volumes, failure to recognize appropriate contexts for protocol initiation, and provider suspicion of "cookbook medicine," with a one-size-fits-all protocol for a varied and heterogeneous patient population. Beyond the persistent uncertainties regarding factors influencing clinician management of ARDS, little is known about changes over time in ventilation practices of nonARDS patients and whether these practices contribute to the pathogenesis of ventilator-induced lung injury and ARDS.

See the Original Study on Page 578

In this issue of Respiratory CARE, Chang et al share the results of a study intended to help answer these and other questions regarding "real world" clinical practice in the ventilator management of patients with and without ARDS. ${ }^{10}$ They performed secondary analysis of an observational study designed to determine early clinical predictors of subsequent ARDS development (the Lung Injury Prevention Study [LIPS] ${ }^{11}$ ), with observational data from 829 patients receiving mechanical ventilation at 22 cen- ters. This study's size places it among the largest of its kind, and enabled the authors to analyze practice in various ICU contexts and to consider inter-center variability. It is thus a welcome contribution to our understanding of the state of contemporary ventilator management.

In addition to its large sample size, the study is strengthened by its authors' access to robust clinical data regarding patients' demographics, indications for mechanical ventilation, and subsequent ICU course. The primary limitation of the study is its restriction to "snapshot" ventilator settings at restricted time points: immediately following intubation and (if applicable) following the subsequent development of ALI/ARDS. Thus the data did not permit the authors to fully assess providers' adherence to the lungprotective ventilation protocol, which demands ongoing reassessment and ventilator titration in response to airway pressures and arterial blood gas results. As a proxy, the authors used their own definition of initial tidal volume under $8 \mathrm{~mL} / \mathrm{kg}$ predicted body weight (PBW) to define patients receiving "low tidal volume ventilation." While this type of proxy is reasonable (and generally necessary) within the confines of "ecological" observational studies such as this, initial ventilator settings in acute respiratory failure are often set before the first arterial blood gas or chest x-ray are available, limiting bedside assessment of ARDS, and (more importantly) may bear little resemblance to ventilator settings in subsequent days. Moreover, in the ARMA protocol, clinicians were permitted 4 hours after patient randomization before the target tidal volume (6 mL/kg PBW) was to be obtained. ${ }^{1}$ It is thus impossible to accurately assess whether this study's patients were receiving "low tidal volume ventilation" (as defined in the ARMA trial) based upon initial ventilator settings alone. These limitations may erase meaningful differences in ventilator management between patients, and should be borne in mind before generalizing the authors' findings.

The authors observed that patients initially intubated while meeting the criteria for ALI/ARDS (enrollment was performed prior to the recent publication of the Berlin definition of ARDS, which removed the designation of $\mathrm{ALI}^{12}$ ) received lower tidal volumes than patients who did not meet the criteria for ALI/ARDS. While this difference is encouraging (and has not always been observed ${ }^{13}$ ), the absolute difference in tidal volumes between these 2 groups 


\section{Mechanical Ventilation of Patients With and Without ARDS: How Far Have We Come?}

(7.96 mL/kg PBW vs $8.45 \mathrm{~mL} / \mathrm{kg}$ PBW) was small. The initial tidal volume of the ALI/ARDS arm is comparable to what has been reported in previous studies, ${ }^{8}$ and is actually statistically insignificant from the initial tidal volume recommended by the most recent iterations of the ARDS Network protocol $(8 \mathrm{~mL} / \mathrm{kg}$ PBW $) .{ }^{14}$ The small difference in tidal volumes between these groups is in large part driven by the initial tidal volume in the nonALI/ARDS group, which is low for non-ARDS patients by historical comparison, ${ }^{15,16}$ and consistent with what has been reported more recently. ${ }^{13}$ Taken with these other studies, this observation raises 2 possibilities: providers may have been moving in recent years toward the use of lower tidal volumes, even in patients who do not have ARDS; and this trend may obscure or erase differences in ventilator-related outcomes such as those measured in the current study. Recall that the "traditional ventilation" strategy in ARMA utilized an initial tidal volume of $12 \mathrm{~mL} / \mathrm{kg}$ PBW, ${ }^{1}$ more than $40 \%$ greater than the median tidal volume observed in the current observational study's nonARDS arm. "Traditional ventilation" is almost certainly a moving target, which makes fair historical comparisons between patients in different studies a challenge, if not impossible.

In their analysis of factors related to initial ventilator settings in patients without ARDS, the authors discovered a remarkable disparity between the likelihood of male and female patients receiving (by their definition) "lower tidal volume ventilation" ( $81.8 \%$ vs $51.1 \%$, respectively). This observation is aligned with those of other recent studies that have demonstrated increased tidal volumes received by female patients, compared to men. ${ }^{4,16}$ As well, as has also been observed in the same studies, this difference disappears when adjusted for height and body mass index. Chang et al speculate (rightly, in my opinion) that many providers unfortunately still often rely on actual body weight rather than PBW in determining tidal volumes, which would predispose heavy and short patients to inappropriately large tidal volumes. Given that obesity is more common among adult women than men in the United States, ${ }^{17}$ and given the on-average shorter stature of women than men, clinician reliance on actual body weight may fully explain this potentially important disparity in critical care delivery. Interestingly, the current study found that male sex was a positive risk factor for subsequent devel-

\footnotetext{
The author has disclosed no conflicts of interest.

Correspondence: Robert P Dickson MD, Division of Pulmonary and Critical Care Medicine, Department of Internal Medicine, University of Michigan, 3916 Taubman Center, Ann Arbor MI 48109. E-mail: robertpdickson@gmail.com.
}

DOI: $10.4187 /$ respcare. 02384 opment of ARDS among non-ARDS patients; thus, if the sex disparity in initial tidal volumes is adversely affecting female patients, any signal of this is being overwhelmed by other contributing factors.

Finally, while we have known for decades from animal modeling and observational human studies that high tidal volume ventilation can provoke lung inflammation, alveolar damage, and pulmonary edema, ${ }^{18,19}$ the question of whether low tidal volume ventilation can prevent subsequent development of ARDS among ventilated patients without ARDS has not been definitively answered. In the Chang et al study, increased initial tidal volume among non-ARDS patients was not significantly associated with subsequent development of ARDS. This finding differs from that of a randomized controlled trial ${ }^{20}$ and several observational series, ${ }^{16,21,22}$ all of which found significant associations between high tidal volumes and subsequent development of ARDS. As mentioned above, the lack of difference seen in the current study may reflect the relatively low (by historic standards) tidal volumes administered to the non-ARDS patients, and should not be generalized in isolation of this context. The general trend in the above-cited literature supports lower tidal volumes for all patients with risk factors for ARDS (though exactly how low remains undetermined). The lack of significant difference in outcomes in the current study may be evidence only that this message has gotten out.

Robert P Dickson MD

Division of Pulmonary and Critical Care Medicine Department of Internal Medicine University of Michigan Ann Arbor, Michigan

\section{REFERENCES}

1. The Acute Respiratory Distress Syndrome Network. Ventilation with lower tidal volumes as compared with traditional tidal volumes for acute lung injury and the acute respiratory distress syndrome. N Engl J Med 2000;342(18),1301-1308.

2. Mcglynn EA, Asch SM, Adams J, Keesey J, Hicks J, DeCristofaro A, Kerr EA. The quality of health care delivered to adults in the United States. N Engl J Med 2003;348(26):2635-2645.

3. Liang L. The gap between evidence and practice. Health Aff (Millwood) 2007;26(2):w119-w121.

4. Han S, Martin GS, Maloney JP, Shanholtz C, Barnes KC, Murray S, Sevransky JE. Short women with severe sepsis-related acute lung injury receive lung protective ventilation less frequently: an observational cohort study. Crit Care 2011;15(6):R262.

5. Kalhan R, Mikkelsen M, Dedhiya P, Christie J, Gaughan C, Lanken $\mathrm{PN}$, et al. Underuse of lung protective ventilation: analysis of potential factors to explain physician behavior. Crit Care Med 2006; 34(2):300-306.

6. Weinert CR, Gross CR, Marinelli WA. Impact of randomized trial results on acute lung injury ventilator therapy in teaching hospitals. Am J Respir Crit Care Med 2003;167(10):1304-1309. 


\section{Mechanical Ventilation of Patients With and Without ARDS: How Far Have We Come?}

7. Young MP, Manning HL, Wilson DL, Mette SA, Riker RR, Leiter $\mathrm{JC}$, et al. Ventilation of patients with acute lung injury and acute respiratory distress syndrome: has new evidence changed clinical practice? Crit Care Med 2004;32(6):1260-1265.

8. Checkley W, Brower R, Korpak A, Thompson BT. Effects of a clinical trial on mechanical ventilation practices in patients with acute lung injury. Am J Respir Crit Care Med 2008;177(11):1215-1222.

9. Rubenfeld GD, Cooper C, Carter G, Thompson BT, Hudson LD. Barriers to providing lung-protective ventilation to patients with acute lung injury. Crit Care Med 2004;32(6):1289-1293.

10. Chang SY, Dabbagh O, Gajic O, Patrawalla A, Elie MC, Talmor D, et al. Contemporary ventilator management in patients with and at risk of ALI/ARDS. Respir Care 2013;58(4):578-588.

11. Gajic O, Dabbagh O, Park PK et al. Early identification of patients at risk of acute lung injury: evaluation of lung injury prediction score in a multicenter cohort study. Am J Respir Crit Care Med 2011; 183(4):462-470

12. ARDS Definition Taskforce; Ranieri VM, Rubenfeld GD, Thompson BT, Ferguson ND, Caldwell E, Fan E, et al. Acute respiratory distress syndrome: the Berlin Definition. JAMA 2012;307(23):2526-2533.

13. Linko R, Okkonen M, Pettilä V, Perttilä J, Parviainen I, Ruokonen E, et al. Acute respiratory failure in intensive care units. FINNALI: a prospective cohort study. Intensive Care Med 2009;35(8):1352-1361.

14. National Institutes of Health; National Heart, Lung and Blood Institute; Acute Respiratory Distress Syndrome Network. NIH NHLBI ARDS clinical network mechanical ventilation protocol summary. http://www.ardsnet.org/system/files/6mlcardsmall_2008update final_JULY2008.pdf. Accessed January 31, 2013.
15. Esteban A, Anzueto A, Alía I, Gordo F, Apezteguía C, Pálizas F, et al. How is mechanical ventilation employed in the intensive care unit? An international utilization review. Am J Respir Crit Care Med 2000;161(5): 1450-1458

16. Gajic O, Dara SI, Mendez JL, Adesanya AO, Festic E, Caples SM, et al. Ventilator-associated lung injury in patients without acute lung injury at the onset of mechanical ventilation. Crit Care Med 2004; 32(9):1817-1824.

17. Flegal KM, Carroll MD, Ogden CL, Curtin LR. Prevalence and trends in obesity among US adults, 1999-2008. JAMA 2010;303(3): 235-241.

18. Greenfield LJ, Ebert PA, Benson DW. Effect of positive pressure ventiation on surface tension properties of lung extracts. Anesthesiology 1964;25:312-316.

19. Dreyfuss D, Saumon G. Ventilator-induced lung injury lessons from experimental studies. Am J Respir Crit Care Med 1998;157(1): 294-323.

20. Determann RM, Royakkers A, Wolthuis EK, Vlaar AP, Choi G, Paulus F, et al. Ventilation with lower tidal volumes as compared with conventional tidal volumes for patients without acute lung injury: a preventive randomized controlled trial. Crit Care 2010;14(1):R1.

21. Gajic O, Frutos-Vivar F, Esteban A, Hubmayr RD, Anzueto A. Ventilator settings as a risk factor for acute respiratory distress syndrome in mechanically ventilated patients. Intensive Care Med 2005; 31(7):922-926.

22. Jia X, Malhotra A, Saeed M, Mark RG, Talmor D. Risk factors for ARDS in patients receiving mechanical ventilation for $>48 \mathrm{~h}$. Chest 2008;133(4):853-861. 